\title{
Alzheimer's Disease Neurodegenerative Biomarkers Are Associated with Decreased Cognitive Function but Not $\beta$-Amyloid in Cognitively Normal Older Individuals
}

\author{
Miranka Wirth, ${ }^{1}$ Cindee M. Madison, ${ }^{1}$ Gil D. Rabinovici, ${ }^{1,2,3}$ Hwamee $0 h,{ }^{1}$ Susan M. Landau, ${ }^{1,2}$ and William J. Jagust ${ }^{1,2}$ \\ ${ }^{1}$ Helen Wills Neuroscience Institute, University of California, Berkeley, Berkeley, California 94720, ${ }^{2}$ Life Sciences Division, Lawrence Berkeley National \\ Laboratory, Berkeley, California 94720, and ${ }^{3}$ Memory and Aging Center, Department of Neurology, University of California, San Francisco, San Francisco, \\ California 94117
}

$\beta$-Amyloid $(\mathrm{A} \beta)$ plaque deposition and neurodegeneration within temporoparietal and hippocampal regions may indicate increased risk of Alzheimer's disease (AD). This study examined relationships between $\mathrm{AD}$ biomarkers of $\mathrm{A} \beta$ and neurodegeneration as well as cognitive performance in cognitively normal older individuals. $A \beta$ burden was quantified in 72 normal older human subjects from the Berkeley Aging Cohort (BAC) using $\left[{ }^{11} \mathrm{C}\right]$ Pittsburgh compound B (PIB) positron emission tomography. In the same individuals, we measured hippocampal volume, as well as glucose metabolism and cortical thickness, which were extracted from a template of cortical AD-affected regions. The three functional and structural biomarkers were merged into a highly AD-sensitive multimodality biomarker reflecting neural integrity. In the normal older individuals, there was no association between elevated PIB uptake and either the single-modality or the multimodality neurodegenerative biomarkers. Lower neural integrity within the AD-affected regions and a control area (the visual cortex) was related to lower scores on memory and executive function tests; the same association was not found with PIB retention. The relationship between cognition and the multimodality AD biomarker was stronger in individuals with the highest PIB uptake. The findings indicate that neurodegeneration occurs within $\mathrm{AD}$ regions regardless of $\mathrm{A} \beta$ deposition and accounts for worse cognition in cognitively normal older people. The impact of neural integrity on cognitive functions is, however, enhanced in the presence of high $\mathrm{A} \beta$ burden for brain regions that are most affected in AD.

\section{Introduction}

There is great interest in detecting and characterizing cognitively normal older individuals with an increased risk of Alzheimer's disease $(\mathrm{AD})$. The hallmark $\mathrm{AD}$ pathology of $\beta$-amyloid $(\mathrm{A} \beta)$ plaque burden and neurodegeneration is seen in a substantial proportion of cognitively normal older people (Morris et al., 2009; Dickerson et al., 2011), suggesting a preclinical stage of AD. Cortical $\mathrm{A} \beta$ deposition has been proposed as the initiating factor in $\mathrm{AD}$ progression (Jack et al., 2010); however, this view is hypothetical and other models have been suggested (Herrup, 2010).

Received Sept. 12, 2012; revised Jan. 21, 2013; accepted Jan. 29, 2013.

Author contributions: M.W. and W.J.J. designed research; M.W., G.D.R., and W.J.J. performed research; M.W., C.M.M., G.D.R., H.O., and S.M.L. analyzed data; M.W. and W.J.J. wrote the paper.

Data collection and sharing for this project was funded by the Alzheimer's Disease Neuroimaging Initiative (ADNI) (National Institutes of Health Grant U01 AG024904). ADNl is funded by the National Institute on Aging, the National Institute of Biomedical Imaging and Bioengineering, and through generous contributions from the following: Abbott; Alzheimer's Association; Alzheimer's Drug Discovery Foundation; Amorfix Life Sciences Ltd. ; AstraZeneca; Bayer HealthCare; BioClinica, Inc.; Biogen Idec Inc.; Bristol-Myers Squibb Company; Eisai Inc.; Elan Pharmaceuticals Inc.; Eli Lilly and Company; F. Hoffmann-La Roche Ltd. and its affiliated company Genentech, Inc.; GE Healthcare; Innogenetics, N.V.; IXICO Ltd.; Janssen Alzheimer Immunotherapy Research and Development, LLC; Johnson and Johnson Pharmaceutical Research and Development LLC; Medpace, Inc.; Merck; Meso Scale Diagnostics, LLC; Novartis Pharmaceuticals Corporation; Pfizer Inc.; Servier; Synarc Inc.; and Takeda Pharmaceutical Company. The Canadian Institutes of Health Research is providing funds to support ADNI clinical sites in Canada. Private sector contributions are facilitated by the Foundation for the National Institutes of Health (www.fnih.org). The grantee organization is the Northern California Institute for Research and Education, and the study is coordinated by the Alzheimer's Disease Cooperative Study at the University of California, San Diego. ADNI data are disseminated by the Laboratory for Neuro Imaging at the University of California, Los Angeles. This research was also supported by NIH
Although preclinical $\mathrm{AD}$ criteria include biomarkers of $\mathrm{A} \beta$ burden and neurodegeneration (Sperling et al., 2011), the relationships between $A \beta$, neuronal damage, and cognition in the normal elderly remain unclear.

Imaged in vivo with [11C] Pittsburgh compound $\mathrm{B}$ (PIB) positron emission tomography (PET) (Klunk et al., 2004), A $\beta$ deposition is found in $20-30 \%$ of cognitively normal older people (Mintun et al., 2006; Quigley et al., 2011). Neurodegenerative AD pathology is known to target circumscribed posterior cortical and hippocampal regions (Perrin et al., 2009). There it can be de-

Grants P30-AG010129, K01-AG030514, AG034570, K23-AG031861, P01-AG1972403, and P50-AG023501, the Alzheimer's Association Grant NIRG-07-59422, the John Douglas French Alzheimer's Foundation, the State of California Department of Health Services Alzheimer's Disease Research Center of California Grant 04-33516, and the Swiss National Science Foundation Grant PA00P1_131515. We thank Suzanne Baker, Grace Tang, and Pia Ghosh for their help in data processing as well as Sylvia Villeneuve and Rik Ossenkoppele for their knowledgeable comments in data discussion. Further, we express our gratitude to Tad Haight for his statistical counsel, Bruce Miller of the University of California, San Francisco (UCSF) Memory and Aging Center for patient referrals, and Michael W. Weiner of the VA Medical Center and UCSF for the MRI scanning of the UCSF Alzheimer's disease patients.

Data used in preparation of this article were obtained from the Alzheimer's Disease Neuroimaging Initiative (ADNI) database (adni.Ioni.ucla.edu). As such, the investigators within the ADNI contributed to the design and implementation of ADNI and/or provided data but did not participate in analysis or writing of this report. A complete listing of ADNI investigators can be found at: http://adni.loni.ucla.edu/wp-content/uploads/how_to_apply/ADNI_Acknowledgement_List.pdf. The authors declare no competing financial interests.

Correspondence should be addressed to Dr. Miranka Wirth, Helen Wills Neuroscience Institute, 132 Barker Hall, \#3190, University of California, Berkeley, Berkeley, CA 94720-3190. E-mail: miranka.wirth@gmail.com.

DOI:10.1523/JNEUROSCI.4409-12.2013

Copyright $\odot 2013$ the authors $\quad 0270-6474 / 13 / 335553-11 \$ 15.00 / 0$ 


\begin{tabular}{|c|c|c|c|c|}
\hline & \multicolumn{2}{|l|}{ Derivation sample } & \multicolumn{2}{|l|}{ Validation sample } \\
\hline & ADNINC & ADNI AD & BACNC & UCSF AD \\
\hline \multicolumn{5}{|l|}{ Demographics } \\
\hline Number of subjects & 39 & 50 & 72 & 19 \\
\hline Age at MRI (years) & $78.0(6.4) 62-94$ & 76.5 (7.4) $59-88$ & $74.9(5.7) 63-88$ & $67.8(9.7)^{* * *} 58-89$ \\
\hline Number of women & $17(44 \%)$ & $20(40 \%)$ & $48(67 \%)$ & $9(47 \%)$ \\
\hline Education (years) & $15.8(2.8) 9-20$ & $14.6(3.1)^{* *} 6-20$ & $17.0(1.9) 12-20$ & $16.4(3.2) 9-22$ \\
\hline \multicolumn{5}{|l|}{ Measurements } \\
\hline$\Delta_{\text {time }}($ MRI-FDG) (years) & $0.1(0.1)$ & $0.1(0.1)$ & $0.2(0.4)$ & $0.4(0.5)$ \\
\hline$\Delta_{\text {time }}($ FDG-A $\beta)$ & florbetapir, 3.1 years ( 2.1 years) & n.a. & $P I B, 0.0 d$ & n.a. \\
\hline & $\operatorname{CSF} A \beta, 23.2 \mathrm{~d}(35.6 \mathrm{~d})$ & n.a. & & n.a. \\
\hline $\begin{array}{l}\Delta_{\text {time }} \text { (NTS-MRI) (years) } \\
\text { Biomarkers }\end{array}$ & $0.1(0.1)$ & $0.1(0.0)$ & $0.3(0.2)$ & $0.3(0.4)$ \\
\hline $\begin{array}{l}\text { Blomarkers } \\
\qquad N \text { of } A \beta \text {-negative }(-) \text { or } A \beta \text {-positive }(+)\end{array}$ & & & & \\
\hline $\begin{array}{l}\text { Not A } \beta \text {-negative }(-) \text { or } A \beta \text {-positive (+) } \\
\text { APOE } \varepsilon 4 \text { carrier }\end{array}$ & $\begin{array}{l}29 \text { florbetapir-, } 10 \text { CSF } A \beta- \\
\quad 6(15 \%)\end{array}$ & $\begin{array}{l}\text { n.a. } \\
36(72 \%)\end{array}$ & $\begin{array}{l}\text { 47 PIB-, } 25 \text { PIB }+ \\
22(31 \%)\end{array}$ & $\begin{array}{l}\text { n.d. } \\
10(53 \%), \text { missing: } 3\end{array}$ \\
\hline \multicolumn{5}{|l|}{ Clinical cognitive status } \\
\hline MMSE & $29.2(1.2)$ & $23.4(2.2)^{*}$ & $28.8(1.4)$ & $20.3(6.6)^{* * *}$ \\
\hline CDR (if available) 0/0.5/1/3 & $39 / 0 / 0$ & $0 / 16 / 34$ & n.a. & 1/5/10/1, missing: 2 \\
\hline
\end{tabular}

Values are given as the mean (SD) and span, if helpful. NTS, Neuropsychological test session; $\Delta$, absolute difference; MMSE, mini mental status examination; CDR, clinical rating scale; n.a., not available. ${ }^{*} p<0.05$; ${ }^{*} p<0.1$ comparison between ADNI NC and ADNI AD; ***p $<0.05$, comparison between BAC NC and UCSF AD.

tected using biomarkers of neuronal function and structure, such as glucose metabolism measured by $[18 \mathrm{~F}]$ fluorodeoxyglucose (FDG) PET (Landau et al., 2011), as well as cortical thickness (Dickerson et al., 2009) and hippocampal volume, both delineated from structural magnetic resonance images (MRIs).

In cognitively normal older individuals, the relationship between $\mathrm{A} \beta$ and cognitive functioning is inconsistent and weak (Aizenstein et al., 2008; Mormino et al., 2009). By contrast, neurodegeneration (as reflected in MRI, FDG PET, and tau biomarkers) seems to be more closely tied to cognitive ability (den Heijer et al., 2006; Dickerson et al., 2011; Desikan et al., 2012). Although atrophy within the hippocampus (Storandt et al., 2009; Rowe et al., 2010) and cortical AD-affected regions (Becker et al., 2011; Chételat et al., 2012) may be related to fibrillar $A \beta$ plaques, other reports have failed to show such associations (Storandt et al., 2012). In previous studies, AD-like neurodegeneration was found in individuals without evidence of cortical $A \beta$ deposition (Dickerson and Wolk, 2012; Jack et al., 2012). These results imply that neurodegenerative abnormalities in $\mathrm{AD}$-affected regions may not be invariably associated with $A \beta$.

Reductions in neural integrity may also be attributed to ageassociated degeneration (Raz and Rodrigue, 2006). This is because AD-related and age-related gray matter atrophy converges on hetero-modal regions (Raz et al., 2004; Fjell et al., 2009). One approach to examine the AD biomarker model in cognitively normal older adults is therefore the definition of biomarkers that have a high enough power to discriminate abnormal (ADrelated) from normal (age-related) neuronal properties on an individual basis.

This study quantified neurodegeneration in cognitively normal older adults using highly sensitive AD biomarkers. First, in a sample of $\mathrm{AD}$ patients and $\mathrm{A} \beta$-negative healthy controls, a multimodality parameter (reflecting neural integrity) was established from single-modality biomarkers (cortical thickness, glucose metabolism, and hippocampal volume) as extracted within ADaffected regions. Second, we assessed whether neurodegeneration within the $\mathrm{AD}$ regions would be associated with both poorer cognitive functions and cortical $A \beta$ deposition.

\section{Materials and Methods}

Study design

The design of the study entailed deriving AD neurodegenerative biomarkers in one group of subjects, and validating them in another. The
Table 2. Demographic information of the BAC NC subsamples

\begin{tabular}{lcc}
\hline & BAC NC PIB- & BAC NC PIB+ \\
\hline Demographics & & \\
$\quad$ Number of subjects & 47 & 25 \\
Age at MRI (years) & $74.9(5.5)$ & $75.0(6.1)$ \\
$\quad$ Number of women & $31(66 \%)$ & $17(68 \%)$ \\
$\quad$ Education (years) & $17.3(1.7)$ & $16.4(2.1)^{*}$ \\
Measurements & & \\
$\quad \Delta_{\text {time }}$ (MRI-FDG) (years) & $0.1(0.4)$ & $0.2(0.5)$ \\
$\Delta_{\text {time }}$ (NTS-MRI) (years) & $0.3(0.2)$ & $0.3(0.2)$ \\
Biomarkers & $11(23 \%)$ & $11(44 \%)$ \\
APOE $\varepsilon 4$ carrier & & \\
Clinical cognitive status & $29.0(1.3)$ & $28.5(1.5)$ \\
$\quad$ MMSE & &
\end{tabular}

sample used for derivation came from the Alzheimer's Disease Neuroimaging Initiative (ADNI) and included a group of $\mathrm{A} \beta$-negative cognitively normal controls (ADNI NC) and a group of patients with mild AD (ADNI AD). The validation sample was comprised of a group of normal older controls from the Berkeley Aging Cohort (BAC NC) and AD patients from the University of California, San Francisco (UCSF AD). Relationships between the neurodegeneration biomarkers, PET measures of $\mathrm{A} \beta$, and cognition were then tested in the BAC NC group.

\section{Subject recruitment}

Written informed consent was obtained from each participant in the study before enrollment in accordance with the local Institutional Review Boards (IRBs) for the ADNI sites, and the University of California, Berkeley, and Lawrence Berkeley National Laboratory (LBNL) for BAC and UCSF subjects. Subject characteristics are provided in Tables 1 and 2.

\section{ADNI NC and AD sample}

The ADNI is a multisite, longitudinal, prospective, natural history study that was launched in 2003 by the National Institute on Aging, the National Institute of Biomedical Imaging and Bioengineering, the Food and Drug Administration, private pharmaceutical companies and non-profit organizations as a public-private partnership. ADNI evaluates serial MRI, PET, and other biomarkers, as well as clinical and neuropsychological markers for the onset and progression of mild cognitive impairment (MCI) and early AD.

Full inclusion/exclusion criteria can be found at www.adni-info.org. In brief, at the time of enrollment, ADNI subjects are between 55 and 90 (inclusive) years old. ADNI NC have MMSE scores 24-30 (inclusive), a clinical dementia rating (CDR) (Morris, 1993) of 0, no signs of depres- 
sion, no memory complaints, and normal memory functions as determined via the Logical Memory II subscale from the Wechsler Memory Scaled-Revised (Wechsler, 1987). Mild AD patients demonstrate a CDR of 0.5 or 1.0, MMSE scores between 20 and 26 (inclusive) and are required to meet NINCDS/ADRDA criteria for probable AD (McKhann et al., 2011).

For this study, a sample of 39 ( 17 females, 22 males) A $\beta$-negative $\mathrm{NC}$ and 50 (20 women) mild AD were selected from the ADNI database. ADNI subjects were included, if they had completed structural 1.5T MRI and FDG PET imaging and cognitive testing. We included only $A \beta$-negative ADNI $\mathrm{NC}$, as defined by $[18 \mathrm{~F}]$ florbetapir-PET or (when florbetapir was not available) CSF A $\beta$ using previously published thresholds (Shaw, 2008). ADNI $\mathrm{NC}$ and $\mathrm{AD}$ groups were matched for age, sex, and education, as well as the time interval between FDG and MRI measurements (Table 1).

\section{BAC NC sample}

The current sample of the BAC NC included a total of 72 (48 females, 24 males) community-dwelling cognitively intact elderly individuals. Eligibility criteria were set to a Geriatric depression scale (GDS) (Yesavage et al., 1982) score $\leq 10$, Mini mental status examination (MMSE) (Folstein et al., 1975) score $\geq 25$, no current neurological and psychiatric illness, normal functions on verbal and visual memory tests (all scores $\geq-1.5 \mathrm{SD}$ of age-adjusted, gender-adjusted, and education-adjusted norms) and age of 60-90 (inclusive) years. All subjects underwent a detailed standardized neuropsychological test session and neuroimaging measurements, all of which were obtained in close temporal proximity (Table 1).

\section{UCSF AD sample}

The sample included 19 ( 9 females, 10 males) patients with mild to severe $\mathrm{AD}$ evaluated at the UCSF Memory and Aging Center. Subjects were required to have FDG and PIB PET images and structural MRI scans obtained on a 1.5T MR system. AD patients met the NINCDS/ADRDA criteria for probable AD (McKhann et al., 2011) and were free of significant comorbid medical, neurologic, or psychiatric illnesses. AD diagnosis was based on a multidisciplinary evaluation.

\section{Cognitive data}

In the BAC NC sample, the dimensionality of the behavioral data were reduced by constructing memory and executive functions composite measures using a two-step procedure. In step 1, domain-sensitive cognitive tests were defined, which were then averaged using $z$ scores in step 2 .

The domain-sensitive tests of memory and executive functions were selected in a procedure that optimized conceptual relevance and suitable measurement characteristics (minimum data loss and mid-range test scores). Domain sensitivity of the cognitive tests was corroborated using a principal component analysis (PCA) with orthogonal (varimax) rotation. The analysis was performed for the test scores of the first neuropsychological test session using a larger sample of the cognitively normal older BAC participants $(\mathrm{N}=303$ at the time of analysis, mean age $=71.5$ (9.0) years, age span $50-96$ years, $\mathrm{N}$ of women $=205(68 \%)$, education $=$ 16.9 (2.1) years, education span 12-20 years, MMSE $=28.8$ (1.3), MMSE span 25-30). Bartlett's test of sphericity indicated that the intercorrelations between cognitive tests were sufficiently large for the PCA. Two factor components were confirmed using Kaiser's criterion of an eigenvalue $>1$ and explained $60.3 \%$ of the total variance in combination.

The memory factor was composed of episodic memory tests, specifically the free recall trials $1-5$ of the California Verbal Learning Test (CVLT) II (Delis et al., 2000), the Logical Memory recall of story A and B (Wechsler, 1997a), and the Visual Reproduction Delayed Recall as well as Recognition (Wechsler, 1997a). The factor measuring executive functions consisted of the Stroop Test (correct naming of printed colors) (Trenerry et al., 1989), the Controlled Oral Word Association Test (Benton et al., 1983), the Trail Making Test Part B (Reitan, 1958) and the Digit Symbol-Coding Test (Wechsler, 1997b). The cognitive tests were combined into the composite measures by converting each test to $z$ scores using the mean and SD of the overall BAC cohort (see above) and averaging them.

Cognitive data used to describe the ADNI participants and the UCSF AD patients were limited to the MMSE and CDR (Table 1).

\section{Neuroimaging data acquisition and preprocessing \\ $M R I$}

ADNINCand AD samples. The high-resolution T1-weighted magnetizationprepared rapid gradient echo (MPRAGE) were obtained at multiple ADNI sites using a standardized acquisition protocol (http://adni.loni.ucla.edu/ research/protocols/mri-protocols/) on General Electric (GE), Siemens, or Philips 1.5T systems. For this study, we used preprocessed MPRAGE scans that had undergone a full set of image correction steps at the time of analysis, including gradient warping (except Philips Scanner), intensity correction, and scaling (available in 33/39 cases) (Jack et al., 2008).

BAC NC samples. MRI scans were acquired at LBNL on a 1.5T Magnetom Avanto system (Siemens) using a 12 channel head coil run in triple mode. Each subject's MPRAGE scans were collected axially with the following measurement parameters: $\mathrm{TR}=2110 \mathrm{~ms}$, TE $=3.58 \mathrm{~ms}$, flip angle $=15^{\circ}$, field of view $=256 \times 256 \mathrm{~mm}$, number of slices $=160$ with a $50 \%$ gap, voxel size $=1 \times 1 \times 1 \mathrm{~mm}^{3}$.

UCSF AD samples. MPRAGE scans were collected coronally via a $1.5 \mathrm{~T}$ Magnetom Vision System (Siemens Medical Systems, Erlangen, Germany) with a quadrature head coil and the following acquisition parameters: $\mathrm{TR}=$ $10 \mathrm{~ms}, \mathrm{TE}=7 \mathrm{~ms}$, flip angle $=15^{\circ}$, voxel size $=1 \times 1 \times 1.5 \mathrm{~mm}^{3}$.

\section{FDG PET}

$A D N I N C$ and $A D$ samples. Multisite FDG PET scans were acquired using standardized FDG acquisition protocols described in detail previously (http://adni.loni.ucla.edu/research/protocols/pet-protocols/). The FDG PET images were collected with the same protocol used for BAC and UCSF subjects. The "raw" FDG PET frames were averaged and processed to yield images with standard orientation, voxel size, and $8 \mathrm{~mm}$ isotropic full-width/ half-maximum (FWHM) resolution (Joshi et al., 2009; Jagust et al., 2010). The preprocessed FDG PET images (standardized orientation, intensity correction, and resolution) were normalized using the pons as the reference region.

$B A C N C$ and UCSF AD samples. FDG PET imaging was performed at LBNL with an ECAT EXACT HR PET scanner (Siemens), $\sim 2 \mathrm{~h}$ after PIB tracer injection. Following 6-10 $\mathrm{mCi}$ of tracer injection, $6 \times 5 \mathrm{~min}$ frames of emission data were collected starting $30 \mathrm{~min}$ after injection. All FDG PET data were reconstructed using an ordered subset expectation maximization algorithm with weighted attenuation. Images were smoothed applying a $4 \times 4 \times 4 \mathrm{~mm}$ Gaussian kernel with scatter correction.

The six "raw" FDG PET frames were aligned to the first frame and averaged using Statistical Parametric Mapping version 8 (SPM8; http://www.fil.ion.ucl.ac.uk/spm). Individual FDG frames were then realigned to the resultant average image and combined to create a singleframe average image. To enhance comparability with the ADNI FDG scans, each subject's average FDG PET image was smoothed to a resolution of $8 \mathrm{~mm}$ FWHM using a Gaussian kernel of $5.5 \mathrm{~mm}$ in plane and 4.0 $\mathrm{mm}$ in the slice direction. The kernel was determined with the same protocol used to create standard resolution images in ADNI (Joshi et al., 2009). The smoothed FDG PET scans were intensity normalized to the pons, as edited from the subcortical FreeSurfer 5.1. parcellation of the native-space MRI scans (Fischl et al., 2002). The choice of reference region was based on evidence indicating preservation of pontine glucose metabolism in AD patients (Minoshima et al., 1995). There was no partial volume correction performed.

\section{PIB PET}

For the BAC NCs, PIB PET scans were collected at LBNL. After $\sim 15 \mathrm{mCi}$ tracer injection into an antecubital vein, dynamic acquisition frames were obtained in the $3 \mathrm{D}$ acquisition mode over a 90 min measurement interval $(4 \times 15 \mathrm{~s}$ frames, $8 \times 30 \mathrm{~s}$ frames, $9 \times 60 \mathrm{~s}$ frames, $2 \times 180 \mathrm{~s}$ frames, $8 \times 300 \mathrm{~s}$ frames, and $3 \times 600 \mathrm{~s}$ frames) after a $10 \mathrm{~min}$ transmission scan. Frames 6-34 were aligned to frame 17 using a two-pass algorithm. Frames $1-5$ were summed and registered to the mean of frames $6-34$, with the alignment parameters applied to each individual frame. The realigned frames corresponding to the first $20 \mathrm{~min}$ of acquisition were averaged and used to coregister the structural MRI to the nativespace PIB PET image. Distribution volume ratios (DVRs) were generated with Logan graphical analysis on the aligned PIB frames using the native- 
space gray matter cerebellum as a reference region (Logan et al., 1996; Klunk et al., 2004; Price et al., 2005), fitting 35-90 min after injection.

For each subject, a global cortical PIB index was derived from the native-space DVR image over frontal (cortical regions anterior to the precentral sulcus), temporal (middle and superior temporal regions), parietal (supramarginal gyrus, inferior/superior parietal lobules, and precuneus), and anterior/posterior cingulate regions-of-interest (ROIs), previously demonstrated to exhibit higher PIB retention in $\mathrm{AD}$ subjects compared with cognitively normal controls (Price et al., 2005). ROIspecific values were extracted from the automated FreeSurfer 5.1 anatomical parcellation using the Desikan-Killiany atlas (Desikan et al., 2006) and combined as a weighted average. There was no partial volume correction performed.

The BAC NC subjects were dichotomized into a high (BAC NC PIB+) and low (BAC NC PIB-) PIB binding status, indicating the presence or absence of abnormal PIB retention, respectively. The specific cutoff score for PIB positivity was two SDs above the mean of the PIB index estimated in an independent group of healthy young adults (Oh et al., 2011; Mormino et al., 2012), yielding a value of 1.08. Demographic information for PIB + and PIB - subgroups of the BAC NC are provided in Table 2.

\section{Neuroimaging data analysis}

MRI

Surface-based cortical thickness maps were estimated from the nativespace MRI scans for each individual using the FreeSurfer 5.1 software package (http://surfer.nmr.mgh.harvard.edu/). The processing pipeline is described previously and will only be explained in brief (Dale et al., 1999; Fischl et al., 1999a). In an automated procedure each MPRAGE scan was bias-field corrected, intensity normalized, and skull stripped using a watershed algorithm (Dale et al., 1999; Segonne et al., 2004). The resulting image was subjected to a white matter segmentation algorithm to define the white/gray matter boundary. After topology correction of the reconstructed white/gray matter border surfaces (Dale et al., 1999; Fischl et al., 2002; Segonne et al., 2004), the pial surfaces were estimated (Fischl and Dale, 2000). A triangular mesh represented the structures of white and gray matter (or pial) surfaces, such that image properties could be mapped to each node (or vertex) of the triangular mesh. Cortical thickness surface maps were derived by calculating the distance between the two surfaces at each vertex across the entire cortex (Fischl and Dale, 2000). The corresponding values were projected onto each subject's reconstructed cortical surface (Fischl et al., 1999a).

\section{FDG PET}

We adopted a procedure previously described (Park et al., 2006). Each subject's preprocessed FDG PET image was coregistered to the MRI scan using an SPM-based coregistration algorithm (Ashburner and Friston, 1997). The coregistered volumetric FDG image was then sampled onto the cortical surface by means of maximum projection. Namely, an intensity profile was created by sampling 10 equal proportions of the FDG PET intensity values between white matter and pial surface at each vertex. The FDG surface maps were created by mapping the maximum intensity value derived from the vertex-wise intensity profile to the reconstructed surface. Maximum projection was chosen because it is more robust to minor registration and MRI segmentation errors (Park et al., 2006).

\section{Derivation and validation of neurodegenerative biomarkers}

We began by defining the single-modality biomarkers of cortical thickness, glucose metabolism (FDG PET), and hippocampal volume (HV) using the $\mathrm{ADNI}$ NC and $\mathrm{AD}$ groups within brain regions most affected by $\mathrm{AD}$. The single-modality biomarkers were then combined into an $\mathrm{AD}$ sensitive multimodality biomarker. To examine whether biomarker effects were stronger within $\mathrm{AD}$-affected regions, a non-AD "control" region was chosen. Similar to a prior study (Dickerson et al., 2009), the bilateral primary visual cortex of the automated FreeSurfer 5.1 DesikanKillany parcellation was used.

\section{Definition of cortical AD-affected regions and}

\section{biomarker extraction}

The individual thickness surface maps were registered to a template surface using a spherical morphing procedure that aligns cortical folding patterns (Fischl et al., 199b). The resulting registration also mapped the FDG surface maps into a common surface space. All surface maps were smoothed by an iterative nearest-neighbor averaging procedure (Fischl et al., 1999b) with a FWHM Gaussian kernel of $15 \mathrm{~mm}$ for cortical thickness and $10 \mathrm{~mm}$ for FDG PET. To validate data quality, we first generated statistical surface maps for FDG PET and cortical thickness using general linear models (GLMs) with the predictors of diagnosis (ADNI NC, ADNI $\mathrm{AD})$ and demeaned covariates of no interest (education and age). The single-modality group contrasts (ADNI NC $>$ ADNI AD) produced maps of $\mathrm{AD}$-related cortical thinning and hypometabolism.

To limit neurodegenerative biomarkers to regions that were affected by both $\mathrm{AD}$-related cortical thinning and hypometabolism, we defined the convergence of cortical thinning and hypometabolism maps. This was done, because our preliminary data analyses indicated that the convergence map comprised a minimum set of cortical regions without reductions in $\mathrm{AD}$-sensitivity and specificity compared with the singlemodality (FDG PET or cortical thickness) statistical surface maps. To extract the convergence map, a binary mask was created from the statistical hypometabolic surface map to spatially restrict the computation of the statistical thinning map using an equivalent GLM. Within each hemisphere, ROIs with an area larger than $300 \mathrm{~mm}^{2}$ were defined in the statistical thinning map and converted to an ROI template of cortical AD regions (or cortical $\mathrm{AD}$ template). For all analyses, the statistical threshold was set to $p<0.00005$ (uncorrected); the equivalent false discovery rate (FDR) threshold is provided.

Using the cortical AD and control templates, cortical thickness and FDG PET were derived for each subject of each sample (ADNI NC, ADNI $\mathrm{AD}, \mathrm{BAC}$ NC, UCSF AD). The templates were mapped onto each subject's native-space cortical thickness and FDG PET surface image using the spherical registration of the MRI scan to the standard brain. For each ROI, mean cortical thickness and FDG PET were estimated. ROI-specific values were averaged and weighted by the number of vertices.

Hippocampal volume was obtained within each hemisphere of the native-space MRI scan using the automated subcortical FreeSurfer 5.1 parcellation and averaged across hemispheres. The HV was adjusted for head size (abbreviated as HVicv) via a regression model including all available subjects. The method removed shared variance with the FreeSurfer 5.1 derived total intracranial volume (ICV); i.e., an estimate that includes brain tissue, CSF-filled, and blood-filled spaces (Mathalon et al., 1993).

Finally, each biomarker was age-adjusted using the ADNI NC sample. Age was regressed on the biomarker of interest within the ADNI NC reference population. The unstandardized regression coefficients estimated age-adjusted residuals for each individual of each sample. The age-adjusted values were $z$-transformed using mean and SD of the ADNI NC sample.

\section{Multimodality biomarker combination}

Using the derivation sample (ADNI NC, ADNI AD) and discriminant function analysis (DFA) the single-modality biomarkers were merged into a continuous multimodality parameter. The DFA determined a linear combination (or function) that best separated (or discriminates) target groups using several predictors (Field, 2005). Diagnostic group (ADNI NC, ADNI AD) was entered as the-dependent variable of interest; the three single-mode age-adjusted and z-transformed biomarkers (cortical thickness, FDG uptake, and HVicv) were fitted as independent variables. The DFA identified one discriminant function (canonial $R^{2}=0.66$ ) that significantly discriminated the diagnostic groups of the derivation sample (Wilk's lamda $(\lambda)$ criterion: $\lambda=0.34, \chi^{2}(3)=91.95$, $p<0.001)$. This function correctly classified $90 \%$ of the ADNI AD patients. Discriminant function scores were estimated for every subject of each sample (ADNI NC, ADNI AD, UCSF AD, BAC NC); scores of $\leq 0$ indicate that the individual would be classified as $\mathrm{AD},>0$ implies normal control classification. The multimodality biomarker of the control region was created by averaging the z-transformed and age-adjusted mean cortical thickness and FDG PET values of the visual cortex.

\section{Validation of the neurodegenerative biomarkers}

The multimodality and single-modality biomarkers were compared between $\mathrm{AD}$ patients and $\mathrm{NC}$ individuals of the derivation and validation 


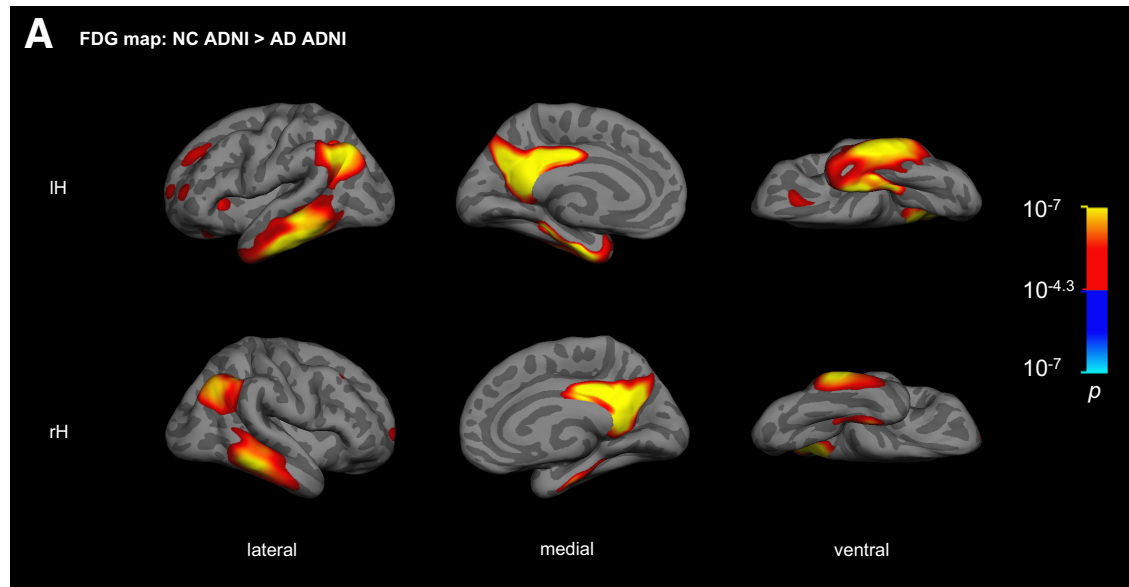

B cThick map: NC ADNI > AD ADNI

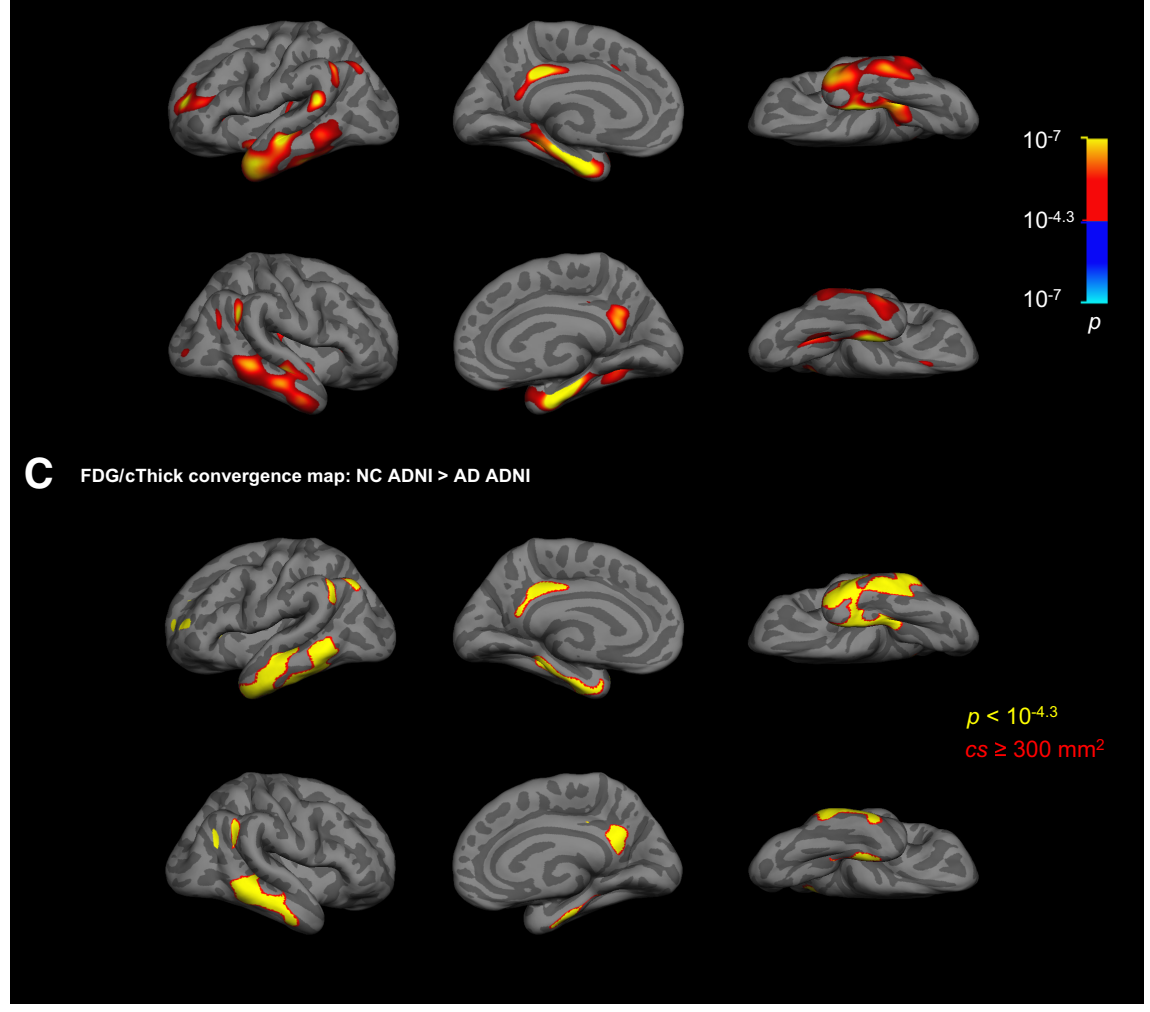

Figure 1. Statistical surface maps. $\boldsymbol{A}-\boldsymbol{C}$, Statistical surface maps $(p<0.00005)$ for FDG PET $(\boldsymbol{A})$, cortical thickness (CThick) $(\boldsymbol{B})$, and the $\mathrm{FDG} / \mathrm{C}$ Thick convergence $(C)$ as derived from the group contrast (ADNI NC $>$ ADNI AD) in the derivation sample. For each surface map, significant clusters are color-coded. Yellow coloring illustrates cortical regions with the most significant AD-related hypometabolism and thinning. The red boundary in Cindicates that the cluster size (cs) exceeded the minimum threshold of $300 \mathrm{~mm}^{2}$.

samples using the $95 \%$ confidence interval (CI) of the difference. If this CI did not include 0 , the group differences were significant.

The discriminatory power of the multimodality and single-modality biomarkers was validated using the BAC NC and UCSF AD groups and the area under the curve (AUC) of the receiver operating characteristic (ROC). The AUC is a measure that represents the ability of a classifier variable to correctly discriminate individuals with and without the disease (Metz, 1978). AUC values may vary between 0.5 (indicating random classification) and 1 (indicating perfect classification).

\section{Application of the neurodegenerative biomarkers in cognitively normal older individuals}

After development and validation of the neurodegenerative biomarkers, they were related to PIB binding status and cross-sectional cognitive performance in cognitively normal older individuals of the BAC NC sample.

\section{Relationships between the} neurodegenerative biomarkers and PIB uptake

For the multimodality biomarker, a univariate analysis of covariance (ANCOVA) was conducted with PIB binding status (BAC NC PIB+, $\mathrm{BACNC} \mathrm{PIB}-$ ) as a predictor. In addition a multivariate ANCOVA was performed to evaluate effects of each single-modality biomarker (cortical thickness, FDG uptake, and HVicv) asdependent variables and dichotomous PIB uptake as independent predictors. In addition, the statistical analyses were restricted to $\mathrm{PIB}+$ subjects, since findings suggest relationships between neurodegenerative biomarkers and $\mathrm{A} \beta$ presence, particularly for individuals with lower levels of CSF A $\beta$ (Fjell et al., 2010c). In the ANCOVA model, the PIB index was fitted as a continuous predictor and the neurodegenerative biomarkers as dependent variables.

To explore the possibility that our results may be impacted by our choice of PIB uptake threshold, we repeated the analysis using a more conservative threshold. Fourteen individuals with highest $\mathrm{A} \beta$ burden (high $\mathrm{BAC} \mathrm{NC}$ $\mathrm{PIB}+$ ) were isolated from the $\mathrm{BAC} \mathrm{NC}$ sample using the iterative outlier approach (Aizenstein et al., 2008). Cases with slight or ambiguous PIB elevation were excluded in these analyses (Mormino et al., 2012).

Unless otherwise stated, model-specific assumptions of homogeneity of covariance matrices and/or regression slopes were confirmed for all ANCOVA models. The statistical threshold was set to $p<0.05$, two-tailed. Pillai's trace values $(\mathrm{V})$ were chosen to evaluate predictor effects. Covariates of age, education, and gender were only included, when a considerable relationship (here $p<0.1$ ) with the dependent variable of interest was empirically indicated.

\section{Relationships between the neurodegenerative biomarker and cognition}

To evaluate relationships between the neurodegenerative biomarker and cognition for the $\mathrm{AD}$-affected and control regions, omnibus multivariate ANCOVA models were performed with cognitive (memory and executive function) performance as dependent variables and the multimodality biomarker as well as PIB uptake status (BACNCPIB +, BAC NC PIB-) as independent predictors. Education and age were fitted as covariates of no interest. Post hoc univariate ANOCA models evaluated predictor effects on either memory performance or executive functions.

\section{Relationships between the neurodegenerative biomarker and high} PIB uptake on cognition

For the multimodality biomarkers of the AD-affected and control regions, omnibus multivariate ANCOVAs were performed with memory performance and executive functions as dependent variables. The models assessed main and interactive effects between dichotomous PIB uptake status (high BAC NC PIB +, BAC NC PIB-) and the multimodality biomarker. Age and education were fitted as categorical covariates (defined by median split) of no interest. In the statistical models with interaction, the multimodality biomarker was z-transformed. The procedure provided a meaningful zero-point, because the main effect of one predic- 
tor represents a "conditional" effect at the value of 0 of the other predictor, when an interaction term is fitted.

The following post hoc analysis was performed to examine whether the interaction effect between PIB uptake and the neurodegeneration biomarkers on cognition were enhanced for the $\mathrm{AD}$ biomarker compared with the control region biomarker. Two partial correlation coefficients were obtained for each PIB uptake group from within group-regression models, one for the AD-affected regions ( $\mathrm{r} 1$ ) and one for the control region (r2). Within each group, the correlation coefficients $(\mathrm{r} 1, \mathrm{r} 2)$ were compared by applying the formula for dependent correlations (Cohen and Cohen, 1975). The significance level was set to $p<0.05$ (one-tailed).

\section{Results}

Definition of cortical AD-affected regions Single-modality comparisons of surfacebased FDG PET and cortical thickness maps for the derivation sample (ADNI NC, ADNI $\mathrm{AD})$ replicated well known regional patterns of hypometabolism and thinning in $\mathrm{AD}$ patients. $\mathrm{AD}$-related hypometabolism $(p<0.00005$ uncorrected, equivalent to FDR $<0.0005$; Fig. $1 A$ ) was mainly present in bilateral temporal (inferior, middle, medial temporal) and inferior parietal (angular gyrus) regions, the frontal (superior) cortex, and the precuneus. AD-related cortical thinning was detected in highly comparable, though somewhat smaller regions $(p<0.00005$ uncorrected, equivalent to FDR $<0.001$; Fig. $1 B)$.

The statistical maps reflecting convergence of AD-related hypometabolism and cortical thinning were extracted in the derivation sample as a set of posterior cortical ROIs ( $p<0.00005$ uncorrected, equivalent to FDR $<0.005$, cs $\geq$ $300 \mathrm{~mm}^{2}$; Fig. 1C). These cortical regions included temporal areas (mostly middle and medial temporal), inferior parietal cortex (angular gyrus) and the posterior cingulate to precuneus regions. The topographical distribution of the $\mathrm{AD}$-affected ROIs was similar across hemispheres, although somewhat different in size.

\section{Validation of the neurodegenerative biomarkers}

There was a clear reduction in the multimodality neurodegenerative biomarker of the $\mathrm{AD}$-affected regions between the $\mathrm{AD}$ patients compared with $\mathrm{NC}$ individuals of the derivation sample (ADNI NC $>$ ADNI AD, 95\% CI of the difference $[2.34,3.19]$ ) and the validation sample (BAC NC > UCSF AD, 95\% CI of the difference $[2.36,3.84]$ ) (Fig. 2A). Similar effects were measured for the single-modality biomarkers of cortical thickness, FDG uptake and HVicv (all 95\% CIs of the difference excluded 0, data displayed in Fig. 2B-D).

Classification accuracy of the single-modality and multimodality biomarkers within the $\mathrm{AD}$ regions was excellent (all $p$ values $<0.001$ ) and similar across the derivation and validation samples. Highest accuracy was measured for the multimodality biomarker (derivation sample: $\mathrm{AUC}=0.98$, validation sample: $\mathrm{AUC}=0.97$ ). For the single-modality biomarkers, highest accuracy was achieved for HVicv (derivation sample: AUC $=0.95$,

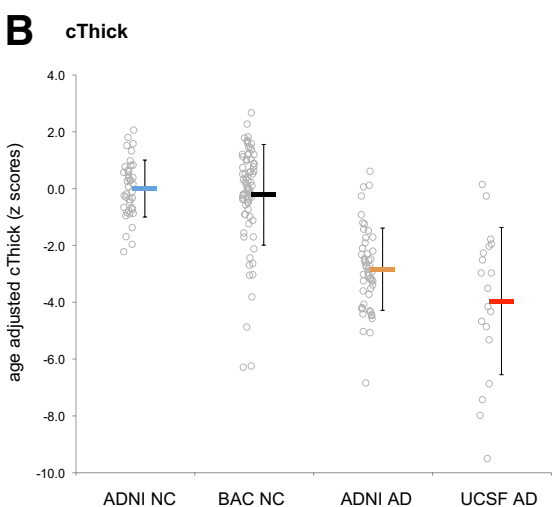

D HVicv

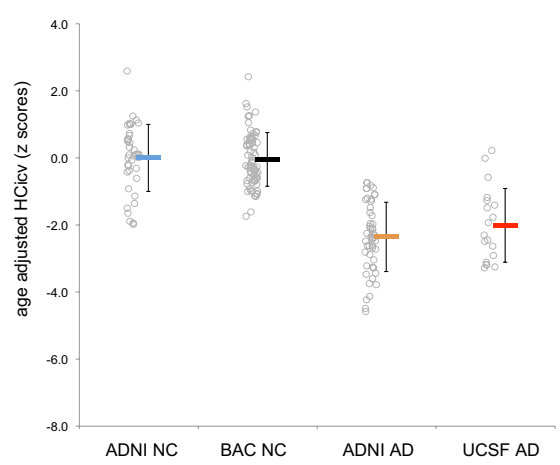

UCSF AD

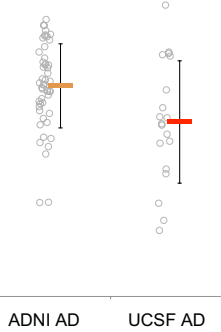

ADNI NC

Figure 2. Validation of the $A D$ neurodegenerative biomarkers. Distribution of the neurodegenerative biomarkers in the derivation (ADNI NC [blue], ADNI AD [orange]) and validation (BAC NC [black], UCSF AD [red]) samples. $\boldsymbol{A}$, The multimodality biomarke plots display group-means and SD for each biomarker; the gray circles indicate individual data points.

validation sample: $\mathrm{AUC}=0.92)$, followed by cortical thickness (derivation sample: AUC $=0.94$, validation sample: AUC $=$ 0.90 ) and FDG PET (derivation sample: $\mathrm{AUC}=0.87$, validation sample: AUC $=0.92$ ).

For the multimodality biomarker of the control region (visual cortex, data not depicted), there was no significant difference between the AD patients and the NC individuals of the derivation sample (ADNI NC $\cong \mathrm{ADNI} A D, 95 \%$ CI of the difference [ -0.10 , $0.50])$. However, there was a difference in the validation sample (BAC NC > UCSF AD, 95\% CI $[0.23,1.04])$, reflecting what is likely more extensive $\mathrm{AD}$ pathology in the UCSF AD patients. The biomarker classification accuracy was random for the derivation sample $(\mathrm{AUC}=0.58, p=0.2)$, and poor, although significant for the validation sample (AUC $=0.70, p<0.01$ ).

\section{Relationships between the neurodegenerative biomarkers and PIB uptake}

In the BAC NC sample, the multimodality biomarker values of $\mathrm{AD}$-affected regions were similar $\left(F_{(1,70)}=0.08, p=0.8\right)$ for $\mathrm{PIB}+$ and $\mathrm{PIB}-$ individuals (Fig. $3 A$ ), as estimated using a univariate ANCOVA. The single-modality biomarkers were also comparable for high and low PIB uptake status $(\mathrm{V}=0.01$, $\left.F_{(3,67)}=0.24, p=0.9\right)$ as confirmed using a multivariate ANCOVA controlling for gender $\left(\mathrm{V}=0.18, F_{(3,67)}=4.8, p<0.01\right)$ (Fig. $3 B-D)$. Likewise, within PIB + individuals there were no significant relationships of the PIB binding index (evaluated as continuous rank transformed and original values) and the $\mathrm{AD}$-sensitive neurodegenerative biomarkers (all $p$ values $\geq 0.1$, data not 

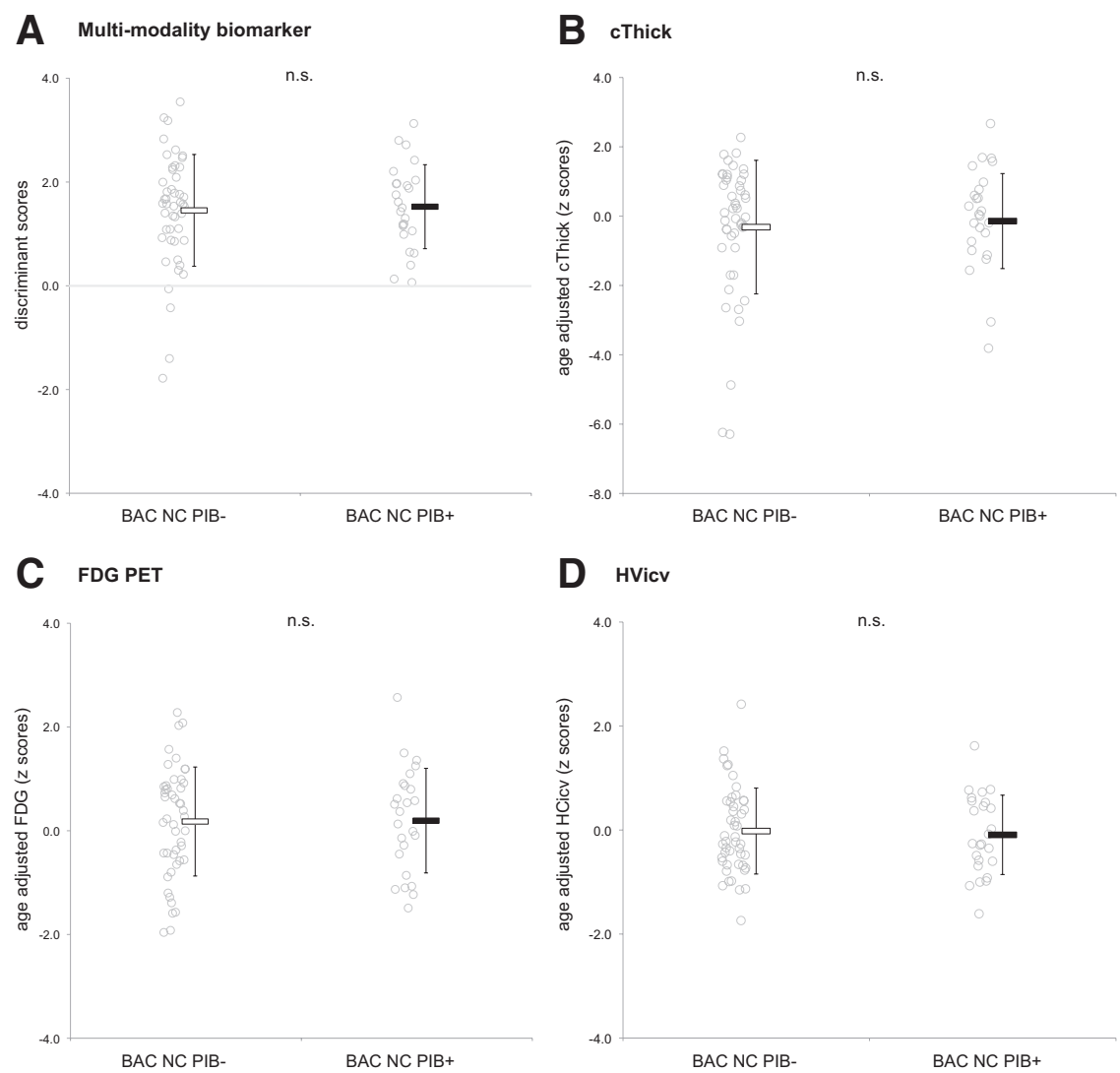

Figure 3. Relationships between the AD neurodegenerative biomarkers and PIB uptake. Distribution of the neurodegenerative biomarkers in PIB negative (BAC NC PIB - [white]) and PIB positive (BAC NC PIB + [solid black]) individuals. A, The multimodality biomarker values were comparable for PIB + and PIB - individuals. The gray line denotes the discriminant cutoff score (values $\leq 0$ indicate $A D$ classification). $\boldsymbol{B}-\boldsymbol{D}$, There were no significant differences in the single-modality biomarkers, i.e., cortical Thickness $(\boldsymbol{B})$, FDG PET ( $\boldsymbol{C}$ and intracranial volume-adjusted hippocampal volume (HVicv) $(\boldsymbol{D})$, between PIB + and PIB - subjects. Error bar plots display group-means and SD for each biomarker; the gray circles indicate individual data points.
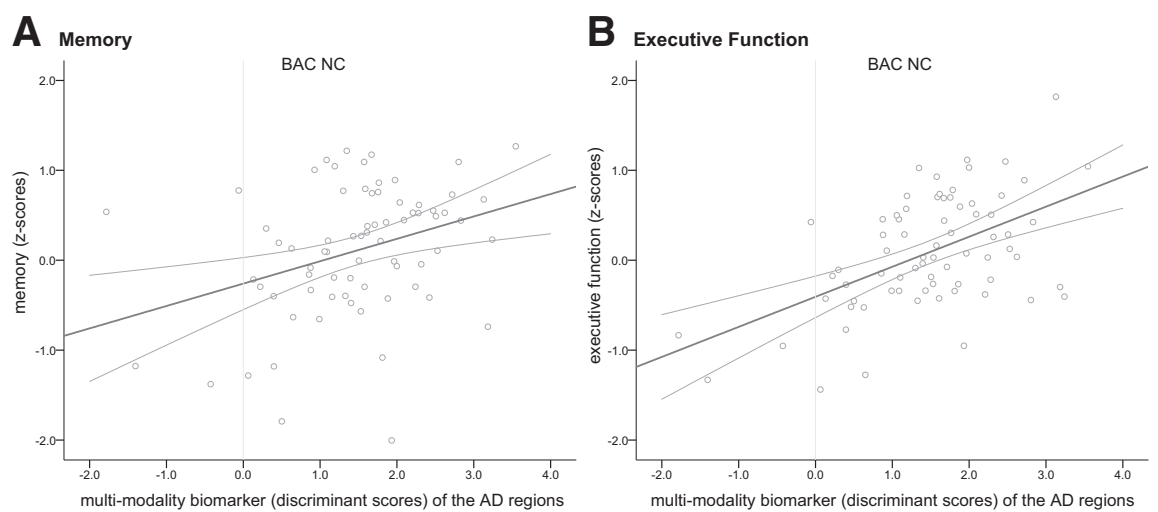

Figure 4. Relationships between the AD neurodegenerative biomarker and cognition. $A, B$, Relationships between the multimodality biomarker and memory performance $(\boldsymbol{A})$ as well as executive functions $(\boldsymbol{B})$ in the BACNC sample. The scatter plots show that lower values of the multimodality biomarker were related to lower memory and executive function. Unadjusted data are depicted; linear trends (dark solid line), 95\% confidence intervals (light solid line), and individual data points (gray circles) are specified.

shown). For the biomarker of the control region, no significant effects of PIB uptake status on the neurodegenerative biomarker were obtained (all $p$ values $\geq 0.2$, data not shown).

\section{Relationships between the neurodegenerative biomarker and cognition}

In the BAC NC sample, the omnibus multivariate ANCOVA demonstrated that the multimodality biomarker of the $\mathrm{AD}$ regions was significantly associated with poorer cognition $\left(\mathrm{V}=0.22, F_{(2,66)}=9.21, p<\right.$ $0.001, \eta 2=0.22)$ accounting for age $(\mathrm{V}=$ $\left.0.20, F_{(2,66)}=7.99, p<0.01\right)$ and education $\left(\mathrm{V}=0.10, F_{(2,66)}=3.79, p<0.05\right)$. Post hoc univariate ANCOVAs corroborated that lower neural integrity explained reductions in memory $\left(F_{(1,67)}=5.06, p<\right.$ $0.05, \eta 2=0.07)$ and executive $\left(F_{(1,67)}=\right.$ 18.26, $p<0.001, \eta 2=0.21)$ functions (Fig. 4).

For the multimodality biomarker of the control region (data not depicted), the ANCOVA model demonstrated that lower values were significantly related to poorer cognition $\left(\mathrm{V}=0.23, F_{(2,66)}=10.04, p<\right.$ $0.001, \eta 2=0.23)$ along with effects of age $\left(\mathrm{V}=0.15, F_{(2,66)}=5.74, p<0.05\right)$ and education $\left(\mathrm{V}=0.18, F_{(2,66)}=7.35, p<0.01\right)$. Post hoc univariate ANCOVAs corroborated that lower biomarker values accounted for reduced memory $\left(F_{(1,67)}=\right.$ 5.63, $p<0.05, \eta 2=0.08)$ and executive $\left(F_{(1,67)}=19.82, p<0.001, \eta 2=0.23\right)$ functions.

There were no effects of dichotomous PIB uptake (all $p$ values $\geq 0.5$ ) on the cognitive measures in any of the statistical models for the AD-affected or the control region.

\section{Relationships between the neurodegenerative biomarker and high PIB uptake on cognition}

The multivariate ANCOVA confirmed the significant main effect of the multimodality biomarker of the $\mathrm{AD}$ regions on cognition $\left(\mathrm{V}=0.39, F_{(2,54)}=17.10, p<\right.$ $0.001, \eta 2=0.39)$. Importantly though, the model also detected a significant interaction between PIB uptake status (high $\mathrm{PIB}+\mathrm{BAC}$ NC, PIB - BAC NC) and the multimodality biomarker $(\mathrm{V}=0.16$, $\left.F_{(2,54)}=5.22, p<0.05, \eta 2=0.16\right)$, controlling for age and education (Fig. 5, top). Post hoc univariate ANCOVA models indicated that the interaction effect was significant for memory $\left(F_{(1,55)}=5.07\right.$, $p<0.05, \eta 2=0.08)$ and executive functions $\left(F_{(1,55)}=9.30, p<0.01, \eta 2=0.15\right)$.

For the control region, the multivariate ANCOVA confirmed the significant main effect of the multimodality biomarker on cognition $\left(\mathrm{V}=0.27, F_{(2,54)}=\right.$ 9.09, $p<0.001, \eta 2=0.27)$, controlling for age and education (Fig. 5, bottom). Importantly though, there was no significant interaction between the PIB uptake status and the neurodegenerative biomarker $(\mathrm{V}=0.02$, $\left.F_{(2,54)}=0.65, p=0.5\right)$.

Post hoc comparisons of the dependent partial correlation coefficients from the neurodegenerative biomarker-cognition relationships within each PIB group (BAC NC PIB+, BAC NC $\mathrm{PIB}-$ ) specified the following: For the high $\mathrm{PIB}+$ individuals, the 
partial correlation coefficient obtained for the $\mathrm{AD}$-affected regions was significantly higher compared with the correlation coefficient of the control region (visual cortex) with regard to memory $\left(r_{\mathrm{AD}}=0.90\right.$, $\left.r_{\text {control }}=0.72, t(11)=2.03, p<0.05\right)$ and executive functions $\left(r_{\mathrm{AD}}=0.82, r_{\text {control }}=\right.$ $0.55, t(11)=2.53, p<0.05)$. For the PIB - group, the partial correlation coefficients, as computed for the AD and control regions, were comparable (all $p$ values $\geq 0.4)$ for memory $\left(r_{\mathrm{AD}}=0.22, r_{\text {control }}=\right.$ $0.27)$ and executive functions $\left(r_{\mathrm{AD}}=0.48\right.$, $\left.r_{\text {control }}=0.48\right)$.

\section{Discussion}

It is increasingly important to identify older individuals who experience cognitive decline and may be in the process of developing AD. Current criteria for preclinical $\mathrm{AD}$ propose that cognitively normal older people, who harbor both amyloid burden and associated neuronal injury, are at an advanced preclinical stage (Jack et al., 2010; Sperling et al., 2011). This study therefore assessed relationships between AD biomarkers of $A \beta$ and neurodegeneration and their relation to cross-sectional cognitive performance in cognitively normal older adults.

There were four important findings: Using an optimized combination of cortical thickness, FDG PET and hippocampal volume, we created a multimodality biomarker with high power to differentiate AD patients from healthy (A $\beta$-negative) older individuals. This $\mathrm{AD}$-sensitive biomarker can be interpreted to capture the presence or loss of neural integrity within regions that are severely affected by $\mathrm{AD}$ pathology. Sampled from cognitively normal older individuals (BAC NC sample), there was no association between the biomarkers of $\mathrm{A} \beta$ burden (measured by the PIB uptake status) and neurodegeneration (measured by the multimodality biomarker). Lower levels of neural integrity within the $\mathrm{AD}$-affected and the control region (visual cortex) were related to poorer cognitive abilities. For the $\mathrm{AD}$ regions, this biomarker-cognition relationship was stronger for individuals with the highest $\mathrm{A} \beta$ burden.

The amyloid cascade model postulates that abnormal $\mathrm{A} \beta$ plaque accumulation is a necessary trigger of $\mathrm{AD}$-typical neurodegeneration (Jack et al., 2010). One can therefore reason that individuals with abnormal neurodegenerative biomarkers would be $\mathrm{A} \beta$-positive. Using a hypothesis-driven approach that defined neural properties within $\mathrm{AD}$-affected regions, we found considerable variability in neural integrity among our normal older individuals, regardless of $\mathrm{A} \beta$ burden. The finding conflicts with the biomarker model, but is consistent with emerging data. A recent study classified the $\mathrm{A} \beta$ and the neurodegenerative biomarker status of normal older people according to criteria for preclinical AD (Jack et al., 2012). In this sample, neurodegenerative $\mathrm{AD}$ biomarker abnormalities were found in a substantial proportion $(23 \%)$ of $A \beta$-negative individuals. In another study, the presence of cortical thinning in $\mathrm{AD}$ signature regions was associated with $\mathrm{AD}$-like $\mathrm{CSF} \mathrm{A} \beta$ in $60 \%$ of subjects, leaving the
B Executive Function
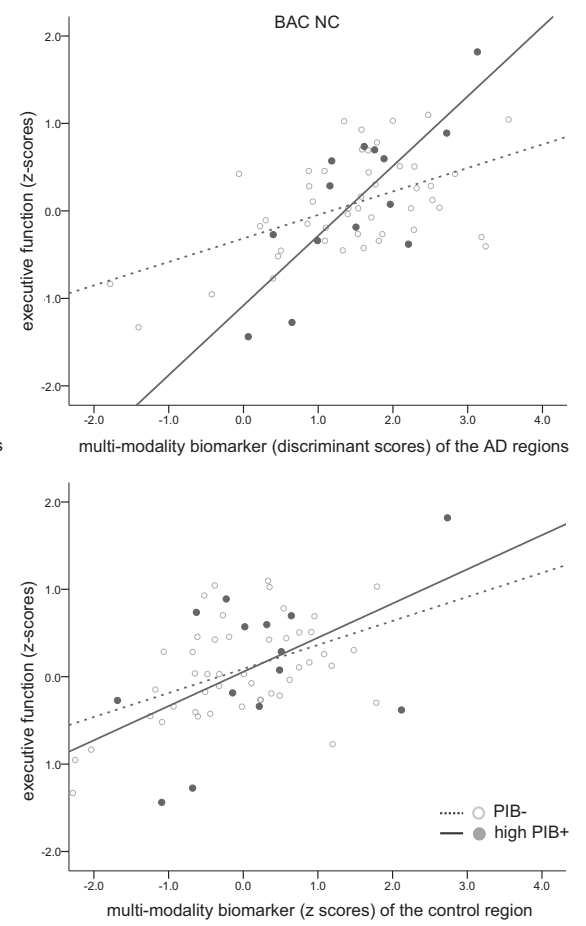

BAC NC
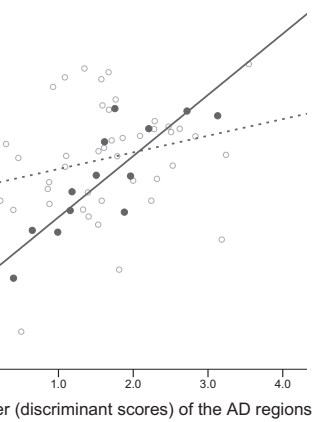

Figure 5. Interaction between the neurodegenerative biomarkers and PIB uptake. $A, B$, Relationships between the multimodality biomarker and memory performance $(\boldsymbol{A})$ as well executive functions $(\boldsymbol{B})$ for the PIB uptake groups of the BAC NC sample. The scatter plots show that for the AD regions (top) the association between the neurodegenerative biomarker and cognitive perfornegative (PIB-) subjects. For the control region, no significant interactions were observed (see text). Unadjusted data are depicted; linear trends (lines) and individual data points (circles) are specified.

high proportion of $40 \%$ of such individuals exhibiting normal CSF A $\beta$ (Dickerson and Wolk, 2012).

Our data and others (Fjell et al., 2010a; Jack et al., 2012) thus converge on the fact that neuronal damage within $\mathrm{AD}$ target regions is non-specific for $\mathrm{A} \beta$ pathology in normal older adults. This suggests that biomarker models and preclinical AD stages proposing $\mathrm{A} \beta$-plaque burden as the initiating factor of $\mathrm{AD}$ typical neurodegeneration could be incorrect (Jack et al., 2010; Sperling et al., 2011). In older people, reductions of neural integrity may be the result of non-AD (pathological) factors that modulate neuronal structure and function over the lifespan. Further, recent demonstrations of trans-neuronal propagation of tau pathology (de Calignon et al., 2012) raise the possibility that the early appearance of entorhinal cortical neurofibrillary tangles could be associated with alterations of function in neocortical neurons projecting to the medial temporal lobe. Thus, in an alternative model to that which proposes $\mathrm{A} \beta$ as the initiating event, tau-related neurodegeneration may arise independently from $\mathrm{A} \beta$ deposition (Mesulam, 1999; Small and Duff, 2008). Scenarios where neurodegenerative abnormalities emerge before or in parallel with $\mathrm{A} \beta$ may converge with theoretical models that posit an initiating role of neuronal injury in the development of $\mathrm{AD}$ (Herrup, 2010).

In our study, lower neural integrity within (but not limited to) $\mathrm{AD}$-affected regions was a close predictor of decreased cognitive abilities. The observation mirrors earlier findings that documented a positive relationship between cognitive abilities and cortical thickness in distributed brain areas (Fjell et al., 2006). A $\beta$ burden itself was a poor predictor of cognitive performance in the 
same individuals corroborating previous inconsistent effects in cross-sectional data (Pike et al., 2007; Aizenstein et al., 2008; Mormino et al., 2009; Oh et al., 2011, 2012). Other studies have highlighted the notion that neuronal injury within $\mathrm{AD}$-affected regions is not benign and may increase the risk for timedependent cognitive decline and $\mathrm{AD}$ conversion in normal older adults (den Heijer et al., 2006; Dickerson et al., 2011). Moreover, in patients with mild cognitive impairment (MCI), medial temporal lobe degeneration without $\mathrm{A} \beta$ abnormalities yielded almost as high risk of $\mathrm{AD}$ conversion than having both $\mathrm{A} \beta$ and neurodegenerative abnormalities (Heister et al., 2011). Our results contribute to these findings by showing that neurodegeneration is associated with poorer cognition regardless of $A \beta$ burden in cognitively intact older adults.

Neuronal injury in $\mathrm{AD}$-affected regions seemed to increase the vulnerability of the brain to late-life $\mathrm{A} \beta$ pathology. This was supported by the interaction between the biomarkers of $A \beta$ deposition and neurodegeneration. Specifically, although $\mathrm{A} \beta$ burden itself was unrelated to the neurodegenerative biomarker and cognitive abilities, worse cognition occurred when $\mathrm{A} \beta$ deposits coincided with lower neural integrity, specifically within the AD regions. The observation agrees with theoretical conceptualizations (Jack et al., 2010; Sperling et al., 2011) and empirical data (Knopman et al., 2012) that denote heightened risk for poor cognitive functions in those individuals that harbor $\mathrm{A} \beta$ burden and neurodegeneration. However, the presence of neurodegeneration without $\mathrm{A} \beta$, and the potentiation of neurodegenerative effects by $\mathrm{A} \beta$, are not consistent with these models. The observation that neurodegeneration and $\mathrm{A} \beta$ are not simply additive suggests that these two processes interact through mechanisms that remain unclear. Similar to our present findings, deleterious $A \beta$ effects on longitudinal cognition change were previously moderated by the presence of tau (Desikan et al., 2012) and signs of neuronal damage within the hippocampus as well as cortical AD regions (Wirth et al., 2013). At the same time, cognitive functioning was maintained in our high $\mathrm{A} \beta$ burden individuals with normal neural integrity, suggesting that $A \beta$ burden per se might not be detrimental (Desikan et al., 2012). The data convey that $\mathrm{AD}$-like neurodegeneration, as emerging from $\mathrm{A} \beta$ independent pathways, could act as a catalyst of $A \beta$-associated effects.

In a broader view, the present findings imply that different (other than $\mathrm{A} \beta$ ) neurodestructive and/or neuroprotective factors cause intersubject variability in neural structure and function, which is relevant to cognitive abilities in advanced age. In particular, age (or aging) itself is associated with cognitive worsening (Hedden and Gabrieli, 2004) and gray matter shrinkage within heteromodal cortices (Raz et al., 2004; Fjell et al., 2009, 2010b). Although the present neurodegenerative biomarkers were adjusted for chronological age, it could be possible that there were residual effects of age that remained. Alternatively, non-AD vascular pathology, even subclinical cerebrovascular risk factors, may cause brain atrophy in normal elderly adults in distributed brain areas (Raz et al., 2004; Leritz et al., 2011). In addition, tau pathology could arise independently from $\mathrm{A} \beta$ in advanced ages, affect neural integrity and interact with $A \beta$ in producing cognitive decline (Small and Duff, 2008). Finally, as a developmental modifier of brain properties, a person's intellectual "baseline" needs to be considered. Intellectual ability in old age is related to intelligence in early life stages (Deary, 2000), where it is associated with enhanced gray matter structure (Hulshoff Pol et al., 2006; Karama et al., 2009; Luders et al., 2009). Thus, a form of brain reserve could be reflected in these measures of brain volume and metabolism.

Our study has several strengths. We created highly sensitive neurodegenerative biomarkers using an independent sample of $\mathrm{AD}$ patients and $\mathrm{A} \beta$-negative, carefully screened cognitively normal older individuals. We further combined single- into multimodality biomarkers using a linear function. Although we had only a small and variable clinical validation group, the results of the validation process coincided with previous observations that multimodality biomarker fusion can increase diagnostic accuracy (Oishi et al., 2011; Zhang et al., 2011). An optimized combination of functional and structural biomarkers has been suggested to increase the power to capture different neuropathological processes in different brain areas. This can be used to improve the differentiation of normal and pathological neuronal properties (Knopman et al., 2012). However, it is important to note that the cross-sectional nature of our study limits interpretation regarding biomarker causality as well as $\mathrm{AD}$ progression.

Together, our data concur with some aspects of the amyloid hypothesis of AD but conflict with others. In agreement with the amyloid hypothesis, we find that neurodegeneration is more strongly associated with cognition than is $A \beta$. However, the interaction between the two processes suggests that neurodegeneration does not simply mediate the effects of $A \beta$ on the brain. The data further conflict with the amyloid hypothesis by showing that $\mathrm{A} \beta$ is not required to develop neurodegeneration within $\mathrm{AD}$-affected regions. The present findings have major bearings on current conceptualizations of aging and preclinical AD.

\section{References}

Aizenstein HJ, Nebes RD, Saxton JA, Price JC, Mathis CA, Tsopelas ND, Ziolko SK, James JA, Snitz BE, Houck PR, Bi W, Cohen AD, Lopresti BJ, DeKosky ST, Halligan EM, Klunk WE (2008) Frequent amyloid deposition without significant cognitive impairment among the elderly. Arch Neurol 65:1509-1517. CrossRef Medline

Ashburner J, Friston K (1997) Multimodal image coregistration and partitioning-a unified framework. Neuroimage 6:209-217. CrossRef Medline

Becker JA, Hedden T, Carmasin J, Maye J, Rentz DM, Putcha D, Fischl B, Greve DN, Marshall GA, Salloway S, Marks D, Buckner RL, Sperling RA, Johnson KA (2011) Amyloid-beta associated cortical thinning in clinically normal elderly. Ann Neurol 69:1032-1042. CrossRef Medline

Benton AL, Hamsher K, Sivan AB (1983) Multilingual aphasia examination. Iowa City, IA: University of Iowa.

Chételat G, Villemagne VL, Villain N, Jones G, Ellis KA, Ames D, Martins RN, Masters CL, Rowe CC (2012) Accelerated cortical atrophy in cognitively normal elderly with high beta-amyloid deposition. Neurology 78:477484. CrossRef Medline

Cohen J, Cohen P (1975) Applied multiple regression/correlation analysis for the behavioral sciences. Hillsdale, NJ: Lawrence Erlbaum.

Dale AM, Fischl B, Sereno MI (1999) Cortical surface-based analysis. I: segmentation and surface reconstruction. Neuroimage 9:179-194. CrossRef Medline

Deary IJ (2000) Looking down on human intelligence: from psychometrics to the brain. New York: Oxford UP.

de Calignon A, Polydoro M, Suárez-Calvet M, William C, Adamowicz DH, Kopeikina KJ, Pitstick R, Sahara N, Ashe KH, Carlson GA, Spires-Jones TL, Hyman BT (2012) Propagation of tau pathology in a model of early Alzheimer's disease. Neuron 73:685-697. CrossRef Medline

Delis DC, Kramer JH, Kaplan E, Ober BA (2000) California verbal learning test, Ed 2. San Antonio, TX: Psychological Corporation.

den Heijer T, Geerlings MI, Hoebeek FE, Hofman A, Koudstaal PJ, Breteler MM (2006) Use of hippocamal and amygdalar volumes on magnetic resonance imaging to predict dementia in cognitively intact elderly people. Arch Gen Psychiatry 63:57-62. CrossRef Medline

Desikan RS, Ségonne F, Fischl B, Quinn BT, Dickerson BC, Blacker D, Buckner RL, Dale AM, Maguire RP, Hyman BT, Albert MS, Killiany RJ (2006) An automated labeling system for subdividing the human cerebral cortex 
on MRI scans into gyral based regions of interest. Neuroimage 31:968980. CrossRef Medline

Desikan RS, McEvoy LK, Thompson WK, Holland D, Brewer JB, Aisen PS, Sperling RA, Dale AM (2012) Amyloid-beta-associated clinical decline occurs only in the presence of elevated P-tau. Arch Neurol 69:709-713. CrossRef Medline

Dickerson BC, Wolk DA (2012) MRI cortical thickness biomarker predicts AD-like CSF and cognitive decline in normal adults. Neurology 78:84-90. CrossRef Medline

Dickerson BC, Bakkour A, Salat DH, Feczko E, Pacheco J, Greve DN, Grodstein F, Wright CI, Blacker D, Rosas HD, Sperling RA, Atri A, Growdon JH, Hyman BT, Morris JC, Fischl B, Buckner RL (2009) The cortical signature of Alzheimer's disease: regionally specific cortical thinning relates to symptom severity in very mild to mild $\mathrm{AD}$ dementia and is detectable in asymptomatic amyloid-positive individuals. Cereb Cortex 19:497-510. CrossRef Medline

Dickerson BC, Stoub TR, Shah RC, Sperling RA, Killiany RJ, Albert MS, Hyman BT, Blacker D, Detoledo-Morrell L (2011) Alzheimer-signature MRI biomarker predicts AD dementia in cognitively normal adults. Neurology 76:1395-1402. CrossRef Medline

Field AP (2005) Discovering statistics using SPSS: and sex, drugs and rock 'n' roll, Ed 2. London: Sage.

Fischl B, Dale AM (2000) Measuring the thickness of the human cerebral cortex from magnetic resonance images. Proc Natl Acad Sci U S A 97: 11050-11055. CrossRef Medline

Fischl B, Sereno MI, Dale AM (1999a) Cortical surface-based analysis. II: inflation, flattening, and a surface-based coordinate system. Neuroimage 9:195-207. CrossRef Medline

Fischl B, Sereno MI, Tootell RB, Dale AM (1999b) High-resolution intersubject averaging and a coordinate system for the cortical surface. Hum Brain Mapp 8:272-284. CrossRef Medline

Fischl B, Salat DH, Busa E, Albert M, Dieterich M, Haselgrove C, van der Kouwe A, Killiany R, Kennedy D, Klaveness S, Montillo A, Makris N, Rosen B, Dale AM (2002) Whole brain segmentation: automated labeling of neuroanatomical structures in the human brain. Neuron 33:341355. CrossRef Medline

Fjell AM, Walhovd KB, Reinvang I, Lundervold A, Salat D, Quinn BT, Fischl B, Dale AM (2006) Selective increase of cortical thickness in highperforming elderly-structural indices of optimal cognitive aging. Neuroimage 29:984-994. CrossRef Medline

Fjell AM, Westlye LT, Amlien I, Espeseth T, Reinvang I, Raz N, Agartz I, Salat DH, Greve DN, Fischl B, Dale AM, Walhovd KB (2009) High consistency of regional cortical thinning in aging across multiple samples. Cereb Cortex 19:2001-2012. CrossRef Medline

Fjell AM, Walhovd KB, Fennema-Notestine C, McEvoy LK, Hagler DJ, Holland D, Brewer JB, Dale AM (2010a) CSF biomarkers in prediction of cerebral and clinical change in mild cognitive impairment and Alzheimer's disease. J Neurosci 30:2088-2101. CrossRef Medline

Fjell AM, Amlien IK, Westlye LT, Stenset V, Fladby T, Skinningsrud A, Eilsertsen DE, Bjørnerud A, Walhovd KB (2010b) CSF biomarker pathology correlates with a medial temporo-parietal network affected by very mild to moderate Alzheimer's disease but not a fronto-striatal network affected by healthy aging. Neuroimage 49:1820-1830. CrossRef Medline

Fjell AM, Walhovd KB, Fennema-Notestine C, McEvoy LK, Hagler DJ, Holland D, Blennow K, Brewer JB, Dale AM (2010c) Brain atrophy in healthy aging is related to CSF Levels of A beta 1-42. Cereb Cortex 20: 2069-2079. CrossRef Medline

Folstein M, Folstein S, McHugh P (1975) "Mini-mental state": a practical method for grading the cognitive state of patients for the clinicians. J Psychiatric Res 12:189-198. CrossRef Medline

Hedden T, Gabrieli JD (2004) Insights into the ageing mind: a view from cognitive neuroscience. Nat Rev Neurosci 5:87-96. CrossRef Medline

Heister D, Brewer JB, Magda S, Blennow K, McEvoy LK (2011) Predicting MCI outcome with clinically available MRI and CSF biomarkers. Neurology 77:1619-1628. CrossRef Medline

Herrup K (2010) Reimagining Alzheimer's disease: an age-based hypothesis. J Neurosci 30:16755-16762. CrossRef Medline

Hulshoff Pol HE, Schnack HG, Posthuma D, Mandl RC, Baare WF, van Oel C, van Haren NE, Collins DL, Evans AC, Amunts K, Burgel U, Zilles K, de Geus E, Boomsma DI, Kahn RS (2006) Genetic contributions to human brain morphology and intelligence. J Neurosci 26:10235-10242. CrossRef Medline
Jack CR Jr, Bernstein MA, Fox NC, Thompson P, Alexander G, Harvey D, Borowski B, Britson PJ, Whitewell JL, Ward C, Dale AM, Felmlee JP, Gunter JL, Hill DL, Killiany R, Schuff N, Fox-Bosetti S, Lin C, Studholme C, DeCarli CS, et al. (2008) The Alzheimer's Disease Neuroimaging Initiative (ADNI): MRI methods. J Magn Reson Imaging 27:685-691. CrossRef Medline

Jack CR Jr, Knopman DS, Jagust WJ, Shaw LM, Aisen PS, Weiner MW, Petersen RC, Trojanowski JQ (2010) Hypothetical model of dynamic biomarkers of the Alzheimer's pathological cascade. Lancet Neurol 9:119-128. CrossRef Medline

Jack CR Jr, Knopman DS, Weigand SD, Wiste HJ, Vemuri P, Lowe V, Kantarci K, Gunter JL, Senjem ML, Ivnik RJ, Roberts RO, Rocca WA, Boeve BF, Petersen RC (2012) An operational approach to National Institute on Aging-Alzheimer's Association criteria for preclinical Alzheimer disease. Ann Neurol 71:765-775. CrossRef Medline

Jagust WJ, Bandy D, Chen K, Foster NL, Landau SM, Mathis CA, Price JC, Reiman EM, Skovronsky D, Koeppe RA (2010) The Alzheimer's Disease Neuroimaging Initiative positron emission tomography core. Alzheimers Dement 6:221-229. CrossRef Medline

Joshi A, Koeppe RA, Fessler JA (2009) Reducing between scanner differences in multi-center PET studies. Neuroimage 46:154-159. CrossRef Medline

Karama S, Ad-Dab'bagh Y, Haier RJ, Deary IJ, Lyttelton OC, Lepage C, Evans AC (2009) Positive association between cognitive ability and cortical thickness in a representative US sample of healthy 6 to 18 year-olds. Intelligence 37:145-155. CrossRef Medline

Klunk WE, Engler H, Nordberg A, Wang Y, Blomqvist G, Holt DP, Bergstrom M, Savitcheva I, Huang GF, Estrada S, Ausen B, Debnath ML, Barletta J, Price JC, Sandell J, Lopresti BJ, Wall A, Koivisto P, Antoni G, Mathis CA, Langstrom B (2004) Imaging brain amyloid in Alzheimer's disease with Pittsburgh Compound-B. Ann Neurol 55:306-319. CrossRef Medline

Knopman DS, Jack CR Jr, Wiste HJ, Weigand SD, Vemuri P, Lowe V, Kantarci K, Gunter JL, Senjem ML, Ivnik RJ, Roberts RO, Boeve BF, Petersen RC (2012) Short-term clinical outcomes for stages of NIA-AA preclinical Alzheimer disease. Neurology 78:1576-1582. CrossRef Medline

Landau SM, Harvey D, Madison CM, Koeppe RA, Reiman EM, Foster NL, Weiner MW, Jagust WJ (2011) Associations between cognitive, functional, and FDG-PET measures of decline in AD and MCI. Neurobiol Aging 32:1207-1218. CrossRef Medline

Leritz EC, Salat DH, Williams VJ, Schnyer DM, Rudolph JL, Lipsitz L, Fischl B, McGlinchey RE, Milberg WP (2011) Thickness of the human cerebral cortex is associated with metrics of cerebrovascular health in a normative sample of community dwelling older adults. Neuroimage 54:2659-2671. CrossRef Medline

Logan J, Fowler JS, Volkow ND, Wang GJ, Ding YS, Alexoff DL (1996) Distribution volume ratios without blood sampling from graphical analysis of PET data. Cereb Blood Flow Metab 16:834-840. Medline

Luders E, Narr KL, Thompson PM, Toga AW (2009) Neuroanatomical correlates of intelligence. Intelligence 37:156-163. CrossRef Medline

Mathalon DH, Sullivan EV, Rawles JM, Pfefferbaum A (1993) Correction for head size in brain-imaging measurements. Psychiatry Res 50:121-139. CrossRef Medline

McKhann GM, Knopman DS, Chertkow H, Hyman BT, Jack CR Jr, Kawas CH, Klunk WE, Koroshetz WJ, Manly JJ, Mayeux R, Mohs RC, Morris JC, Rossor MN, Scheltens P, Carrillo MC, Thies B, Weintraub S, Phelps CH (2011) The diagnosis of dementia due to Alzheimer's disease: recommendations from the National Institute on Aging-Alzheimer's Association workgroups on diagnostic guidelines for Alzheimer's disease. Alzheimers Dement 7:263-269. CrossRef Medline

Mesulam MM (1999) Neuroplasticity failure in Alzheimer's disease: bridging the gap between plaques and tangles. Neuron 24:521-529. CrossRef Medline

Metz CE (1978) Basic principles of ROC analysis. Sem Nucl Med 8:283-298. CrossRef Medline

Minoshima S, Frey KA, Foster NL, Kuhl DE (1995) Preserved pontine glucose metabolism in Alzheimer disease: a reference region for functional brain image (PET) analysis. J Comput Assist Tomogr 19:541-547. CrossRef Medline

Mintun MA, Larossa GN, Sheline YI, Dence CS, Lee SY, Mach RH, Klunk WE, Mathis CA, DeKosky ST, Morris JC (2006) [11C]PIB in a nondemented population: potential antecedent marker of Alzheimer disease. Neurology 67:446-452. CrossRef Medline 
Mormino EC, Kluth JT, Madison CM, Rabinovici GD, Baker SL, Miller BL, Koeppe RA, Mathis CA, Weiner MW, Jagust WJ (2009) Episodic memory loss is related to hippocampal-mediated beta-amyloid deposition in elderly subjects. Brain 132:1310-1323. CrossRef Medline

Mormino EC, Brandel MG, Madison CM, Rabinovici GD, Marks S, Baker SL, Jagust WJ (2012) Not quite PIB-positive, not quite PIB-negative: slight PIB elevations in elderly normal control subjects are biologically relevant. Neuroimage 59:1152-1160. CrossRef Medline

Morris JC (1993) The Clinical Dementia Rating (CDR): current version and scoring rules. Neurology 43:2412-2414. Medline

Morris JC, Roe CM, Grant EA, Head D, Storandt M, Goate AM, Fagan AM, Holtzman DM, Mintun MA (2009) Pittsburgh compound B imaging and prediction of progression from cognitive normality to symptomatic Alzheimer disease. Arch Neurol 66:1469-1475. CrossRef Medline

Oh H, Mormino EC, Madison C, Hayenga A, Smiljic A, Jagust WJ (2011) Beta-Amyloid affects frontal and posterior brain networks in normal aging. Neuroimage 54:1887-1895. CrossRef Medline

Oh H, Madison C, Haight TJ, Markley C, Jagust WJ (2012) Effects of age and beta-amyloid on cognitive changes in normal elderly people. Neurobiol Aging 33:2746-2755. CrossRef Medline

Oishi K, Akhter K, Mielke M, Ceritoglu C, Zhang J, Jiang H, Li X, Younes L, Miller MI, van Zijl PC, Albert M, Lyketsos CG, Mori S (2011) Multimodal MRI analysis with disease-specific spatial filtering: initial testing to predict mild cognitive impairment patients who convert to Alzheimer's disease. Front Neurol 2:54. CrossRef Medline

Park HJ, Lee JD, Chun JW, Seok JH, Yun M, Oh MK, Kim JJ (2006) Cortical surface-based analysis of 18F-FDG PET: measured metabolic abnormalities in schizophrenia are affected by cortical structural abnormalities. Neuroimage 31:1434-1444. CrossRef Medline

Perrin RJ, Fagan AM, Holtzman DM (2009) Multimodal techniques for diagnosis and prognosis of Alzheimer's disease. Nature 461:916-922. CrossRef Medline

Pike KE, Savage G, Villemagne VL, Ng S, Moss SA, Maruff P, Mathis CA, Klunk WE, Masters CL, Rowe CC (2007) Beta-amyloid imaging and memory in non-demented individuals: evidence for preclinical Alzheimer's disease. Brain 130:2837-2844. CrossRef Medline

Price JC, Klunk WE, Lopresti BJ, Lu X, Hoge JA, Ziolko SK, Holt DP, Meltzer CC, DeKosky ST, Mathis CA (2005) Kinetic modeling of amyloid binding in humans using PET imaging and Pittsburgh Compound-B. Cereb Blood Flow Metab 25:1528-1547. CrossRef Medline

Quigley H, Colloby SJ, O'Brien JT (2011) PET imaging of brain amyloid in dementia: a review. Int J Geriatr Psychiatry 26:991-999. CrossRef Medline

Raz N, Rodrigue KM (2006) Differential aging of the brain: patterns, cognitive correlates and modifiers. Neurosci Biobehav Rev 30:730-748. CrossRef Medline

Raz N, Gunning-Dixon F, Head D, Rodrigue KM, Williamson A, Acker JD (2004) Aging, sexual dimorphism, and hemispheric asymmetry of the cerebral cortex: replicability of regional differences in volume. Neurobiol Aging 25:377-396. CrossRef Medline
Reitan RM (1958) Validity of the trailmaking test as an indication of organic brain damage. Percept Mot Skills 8:271-276. CrossRef

Rowe CC, Ellis KA, Rimajova M, Bourgeat P, Pike KE, Jones G, Fripp J, Tochon-Danguy H, Morandeau L, O’Keefe G, Price R, Raniga P, Robins P, Acosta O, Lenzo N, Szoeke C, Salvado O, Head R, Martins R, Masters CL, et al. (2010) Amyloid imaging results from the Australian Imaging, Biomarkers and Lifestyle (AIBL) study of aging. Neurobiol Aging 31: 1275-1283. CrossRef Medline

Ségonne F, Dale AM, Busa E, Glessner M, Salat D, Hahn HK, Fischl B (2004) A hybrid approach to the skull stripping problem in MRI. Neuroimage 22:1060-1075. CrossRef Medline

Shaw LM (2008) PENN biomarker core of the Alzheimer's Disease Neuroimaging Initiative. Neurosignals 16:19-23. CrossRef Medline

Small SA, Duff K (2008) Linking A beta and Tau in late-onset Alzheimer's disease: a dual pathway hypothesis. Neuron 60:534-542. CrossRef Medline

Sperling RA, Aisen PS, Beckett LA, Bennett DA, Craft S, Fagan AM, Iwatsubo T, Jack CR Jr, Kaye J, Montine TJ, Park DC, Reiman EM, Rowe CC, Siemers E, Stern Y, Yaffe K, Carrillo MC, Thies B, Morrison-Bogorad M, Wagster MV, Phelps CH (2011) Toward defining the preclinical stages of Alzheimer's disease: recommendations from the National Institute on Aging-Alzheimer's Association workgroups on diagnostic guidelines for Alzheimer's disease. Alzheimers Dement 7:280-292. CrossRef Medline

Storandt M, Mintun MA, Head D, Morris JC (2009) Cognitive decline and brain volume loss as signatures of cerebral amyloid-beta peptide deposition identified with Pittsburgh compound B: cognitive decline associated with Abeta deposition. Arch Neurol 66:1476-1481. CrossRef Medline

Storandt M, Head D, Fagan AM, Holtzman DM, Morris JC (2012) Toward a multifactorial model of Alzheimer disease. Neurobiol Aging 33:2262-2271. CrossRef Medline

Trenerry MR, Crosson B, DeBoe J, Leber WR (1989) Stroop Neuropsychological Screening Test Manual. Odessa, FL: Psychological Assessment Resources.

Wechsler D (1987) Manual for Wechsler memory scale, revised. San Antonio, TX: Psychological Corporation.

Wechsler D (1997a) Wechsler memory scale: administration and scoring manual, Ed 3. San Antonio, TX: Psychological Corporation.

Wechsler D (1997b) Wechsler adult intelligence scale: administration and scoring manual, Ed 3. San Antonio, TX: Psychological Corporation.

Wirth M, Oh H, Mormino EC, Markley C, Landau SM, Jagust W (2013) The effect of beta-amyloid on longitudinal cognitive decline is modulated by neural integrity in cognitively normal elderly. Alzheimers Dement, in press.

Yesavage JA, Brink TL, Rose TL, Lum O, Huang V, Adey M, Leirer VO (1982) Development and validation of a geriatric depression screening scale: a preliminary report. J Psychiatric Res 17:37-49. CrossRef Medline

Zhang D, Wang Y, Zhou L, Yuan H, Shen D (2011) Multimodal classification of Alzheimer's disease and mild cognitive impairment. Neuroimage 55:856-867. CrossRef Medline 